\title{
@creative
}

ISSN 2590-9770

The Art of Discrete and Applied Mathematics 4 (2021) \#P2.05

https://doi.org/10.26493/2590-9770.1365.884

(Also available at http://adam-journal.eu)

\section{Infinite Paley graphs}

\author{
Gareth A. Jones* \\ Mathematical Sciences, University of Southampton, Southampton SO17 1BJ, UK
}

Received 15 March 2020, accepted 8 June 2020, published online 10 March 2021

\begin{abstract}
Infinite analogues of the Paley graphs are constructed, based on uncountably many locally finite fields. By using character sum estimates due to Weil, they are shown to be isomorphic to the countable random graph of Erdős, Rényi and Rado.
\end{abstract}

Keywords: Paley graph, random graph, universal graph, quadratic residue, character sum.

Math. Subj. Class.: 05C63, 03C13, 03C15, 05C80, 05E18, 11L40, 12E20, 20B25, $20 B 27$.

\section{Introduction}

In 1963 Erdős and Rényi [14] described two constructions of graphs which have subsequently become well-known and well-understood parts of the landscape of graph theory. One construction gave a countably infinite family of finite graphs, defined deterministically, which later became known as the Paley graphs $P(q)$. The other gave a single countably infinite graph $R$ (or more precisely an uncountable family of mutually isomorphic countably infinite graphs), defined randomly and later variously named after Erdős, Rényi and Rado, who gave an alternative construction in [24] the following year. It is perhaps surprising that in the following half-century and more, a strong connection between these very different graphs seems to have received little notice, except in the world of model theory (see [20, Examples 1.3.6 and 1.8.3]), though there are hints to be found in papers such as [2,3]. Perhaps this lacuna is less surprising when one realises that an essential ingredient in this connection comes from algebraic geometry, namely Weil's estimate for character-sums, used in his proof of the Riemann hypothesis for curves over finite fields.

The first aim of this paper is give a more combinatorial explanation of this connection by constructing, for each odd prime $p$, infinite analogues of the Paley graphs, defined over uncountably many locally finite fields of characteristic $p$, and its second aim is to show that these graphs are all isomorphic to $R$. The finite and infinite Paley graphs are described in

\footnotetext{
*The author is grateful to Peter Cameron, Dugald Macpherson, Yaokun Wu and the referees for some very helpful comments.

E-mail address: G.A.Jones@maths.soton.ac.uk (Gareth A. Jones)
} 
Sections 2 and 3, and $R$ is described in Section 4. The isomorphism is proved in Section 5, with remarks on the proof in Section 6. The automorphism groups of these finite and infinite graphs are compared in Section 7, and the construction and the isomorphism with $R$ are extended in Section 8 to the generalised Paley graphs introduced by Lim and Praeger in [19]. The paper [14] is revisited in Section 9.

\section{The Paley graphs and their inclusions}

For each prime power $q=p^{e} \equiv 1 \bmod (4)$ the Paley graph $P(q)$ has as its vertex set the field $\mathbb{F}_{q}$ of $q$ elements, with vertices $x$ and $y$ adjacent if and only if $x-y$ is a quadratic residue (non-zero square) in $\mathbb{F}_{q}$. It is an undirected strongly regular graph with parameters $v=q$ (the number of vertices), $k=(q-1) / 2$ (their common valency), $\lambda=(q-5) / 4$ and $\mu=(q-1) / 4$ (the number of common neighbours of two adjacent or non-adjacent vertices). See [4] for further basic properties of the Paley graphs.

These graphs were introduced, not (as is often asserted) by Paley [23] in 1933, but in the case $e=1$ in 1962 by Sachs [25], as examples of self-complementary graphs, and in the general case $e \geq 1$ in 1963 by Erdős and Rényi [14, §1], as part of their study of asymmetric graphs. Neither paper attached a name to these graphs; they appear to have been named around 1970, no doubt by analogy with Paley designs (see [12]) which, like the orthogonal matrices constructed by Paley in [23], are based on properties of quadratic residues in finite fields. See [18] for a discussion of the history of these graphs.

A finite field $\mathbb{F}_{q}$ is a subfield of $\mathbb{F}_{q^{\prime}}$ if and only if $q^{\prime}$ is a power $q^{f}$ of $q$, in which case the subfield is unique, consisting of the solutions of the equation $x^{q}=x$. In this case, if $q \equiv 1 \bmod (4)$ then $q^{\prime} \equiv 1 \bmod (4)$, so we have Paley graphs $P(q)$ and $P\left(q^{\prime}\right)$. Clearly each quadratic residue in $\mathbb{F}_{q}$ is also a quadratic residue in $\mathbb{F}_{q^{\prime}}$, so $P(q)$ is a subgraph of $P\left(q^{\prime}\right)$. If $f$ is even then every element of $\mathbb{F}_{q}$ has a square root in $\mathbb{F}_{q^{\prime}}$ (in fact, in the quadratic subfield $\mathbb{F}_{q^{2}} \subseteq \mathbb{F}_{q^{\prime}}$ ) so the subgraph of $P\left(q^{\prime}\right)$ induced by $P(q)$ is a complete graph $K_{q}$. However, if $f$ is odd then an element of $\mathbb{F}_{q}$ has a square root in $\mathbb{F}_{q^{\prime}}$ if and only if it has one in $\mathbb{F}_{q}$, so the induced subgraph is simply $P(q)$, that is, $P(q)$ is a full subgraph of $P\left(q^{\prime}\right)$.

\section{Infinite Paley graphs}

Let $E$ be any set of odd $e \in \mathbb{N}$ which is closed under taking divisors and least common multiples. For any prime $p \equiv 1 \bmod (4)$ let

$$
\mathbb{F}_{p^{E}}:=\bigcup_{e \in E} \mathbb{F}_{p^{e}},
$$

the direct limit of the direct system of fields $\mathbb{F}_{p^{e}}$ for $e \in E$ and inclusions between them. This is a subfield of the algebraic closure $\overline{\mathbb{F}}_{p}$ of $\mathbb{F}_{p}$, infinite if and only if $E$ is, and locally finite in the sense that each finite subset is contained in a finite subfield. The finite subfields of $\mathbb{F}_{p^{E}}$ are just the fields $\mathbb{F}_{p^{e}}$ for $e \in E$, so distinct sets $E$ determine distinct (and nonisomorphic) fields $\mathbb{F}_{p^{E}}$. There are uncountably many sets $E$ satisfying the above conditions (consider, for example, the set of integers $e$ whose prime factors all belong to a given set of odd primes), so for each $p$ we obtain uncountably many non-isomorphic fields $\mathbb{F}_{p^{E}}$.

Now let us define

$$
P\left(p^{E}\right):=\bigcup_{e \in E} P\left(p^{e}\right)
$$


the direct limit of the Paley graphs $P\left(p^{e}\right)$ for $e \in E$, with respect to the embeddings $P\left(p^{e}\right) \subseteq P\left(p^{e^{\prime}}\right)$ where $e$ divides $e^{\prime}$ in $E$. By our remarks in Section 2, each $P\left(p^{e}\right)$ is a full subgraph of $P\left(p^{E}\right)$. If $E$ is finite then $E$ is just the set of all divisors of $l:=\operatorname{lcm}(E)$, so that $P\left(p^{E}\right)$ is just another Paley graph $P\left(p^{l}\right)$. We will therefore assume from now on that $E$ is infinite, in which case we will call $P\left(p^{E}\right)$ an infinite Paley graph (see [20, Example 1.8.3] for a similar construction by Macpherson and Steinhorn, though the exponents $e=2^{i}$ used there should be replaced with odd integers). In Section 5 we will use the fact that if, as assumed, $E$ is infinite then each $e \in E$ divides infinitely many elements $e^{\prime} \in E$ : only finitely many elements of $E$ can have a given least common multiple $e^{\prime}$ with $e$, so $e^{\prime}$ takes infinitely many distinct values, all divisible by $e$. Thus each finite subfield $\mathbb{F}_{p^{e}}$ of $\mathbb{F}_{p^{E}}$ or Paley subgraph $P\left(p^{e}\right)$ of $P\left(p^{E}\right)$ is contained in infinitely many others.

In the same way as this one can construct infinite Paley graphs $P\left(p^{2 E}\right)$ for primes $p \equiv-1 \bmod (4)$ as unions of Paley subgraphs $P\left(p^{2 e}\right)$ where $e$ is odd.

Although they are constructed from uncountably many mutually non-isomorphic fields, these infinite Paley graphs $P\left(p^{E}\right)$ and $P\left(p^{2 E}\right)$ are all isomorphic to each other. In fact, we shall prove:

Theorem 3.1. Each infinite Paley graph $P\left(p^{r E}\right)$ for $r=1,2$ is isomorphic to the random graph $R$.

\section{The countable random graph}

The countable random graph, or universal graph $R$ was introduced by Erdős and Rényi [14, $\S 3]$ in 1963 and Rado [24] in 1964. For details of its properties see [6, 7, 8] or [13, Section 9.6], and for some recent generalisations see [1, 15]. Theorem 3.1 should not be as surprising as it might at first appear, since in a sense we shall now explain 'almost all' countably infinite graphs are isomorphic to $R$. (However, the isomorphism class containing $R$ is very far from being random, in the colloquial sense of being typical, since it is just one among uncountably many isomorphism classes of countably infinite graphs.)

Erdős and Rényi showed that if a graph $\Gamma$ has a countably infinite vertex set, and its edges are chosen randomly, then with probability 1 it has the following property $U$ : given any two disjoint finite sets $A$ and $B$ of vertices of $\Gamma$, there is a vertex which is a neighbour of each vertex in $A$ and a non-neighbour of each vertex in $B$. They used this to show that $\Gamma$ is symmetric (has a non-identity automorphism) with probability 1 (by contrast with the finite case, where they showed that a random graph of order $n$ is symmetric with probability approaching 0 as $n \rightarrow \infty$ ). In fact, a similar argument shows that any two countably infinite graphs with property $U$ are isomorphic: one can construct an isomorphism between them by using $U$ to extend, by a back-and-forth argument, one vertex at a time, any isomorphism between finite induced subgraphs, such as a single vertex in each of them. (See, for example, [21, Theorem 2.4.2], which in the language of model theory shows that the theory of graphs with property $U$ is satisfiable and $\aleph_{0}$-categorical, and hence complete and decidable.) Thus any two graphs $\Gamma$ constructed randomly as above are isomorphic with probability 1 .

As a model of $R$ one can therefore take any countably infinite graph with property $U$. For instance, Rado [24] constructed a 'universal graph', in which every countable graph is embedded as an induced subgraph, by using the vertex set $V=\mathbb{N}$ (including 0), with vertices $x<y$ adjacent if and only if $2^{x}$ appears in the binary representation of $y$ as a sum of distinct powers of 2 ; this easily implies property $U$. 
For an alternative model of $R$, let the vertex set $V$ be the (countably infinite) set of all primes $p \equiv 1 \bmod (4)$, and define distinct vertices $p$ and $q$ to be adjacent if and only if $q$ is a quadratic residue mod $(p)$, that is, the Legendre symbol $\left(\frac{q}{p}\right)=1$. By quadratic reciprocity, which states that $\left(\frac{p}{q}\right)\left(\frac{q}{p}\right)=1$ for primes $p, q \equiv 1 \bmod (4)$, this is a symmetric relation, so it defines an undirected graph. To show that this graph has property $U$, given disjoint finite subsets $A$ and $B$ of $V$, for each prime $a \in A$ choose an integer $n_{a}$ which is a quadratic residue mod $(a)$, and for each prime $b \in B$ choose an integer $n_{b}$ which is a non-residue mod $(b)$. By the Chinese Remainder Theorem, the simultaneous congruences $n \equiv 1 \bmod (4)$ and $n \equiv n_{c} \bmod (c)$ for all $c \in C:=A \cup B$ have a unique solution $n$ $\bmod (d)$ where $d=4 \prod_{c \in C} c$, and by a theorem of Dirichlet this congruence class contains a prime (infinitely many, in fact). This gives a vertex in $V$ adjacent to all the vertices $a \in A$ and to none of the vertices $b \in B$, as required.

\section{Proof of Theorem 3.1}

In order to prove Theorem 3.1 it is sufficient to prove that the infinite Paley graphs $P\left(p^{r E}\right)$ all have property $U$. Any pair of disjoint finite sets of vertices $A$ and $B$ are contained in some finite subfield $\mathbb{F}_{q}$ of $\mathbb{F}_{p^{r E}}$. As noted earlier, since $E$ is infinite $\mathbb{F}_{q}$ is contained in finite subfields $\mathbb{F}_{q^{\prime}}\left(q^{\prime}=q^{f}\right)$ of $\mathbb{F}_{p^{r E}}$ for infinitely many odd $f$. It is therefore sufficient for us to show that for all sufficiently large powers $q^{\prime}$ of $q$ there is an element $x \in \mathbb{F}_{q^{\prime}}$ such that $x-a$ is a quadratic residue in $\mathbb{F}_{q^{\prime}}$ for all $a \in A$ and $x-b$ is a non-residue in $\mathbb{F}_{q^{\prime}}$ for all $b \in B$.

We will adapt an argument used by Blass, Exoo and Harary [2] to obtain a similar result concerning the family of Paley graphs $P(p)$ for primes $p \equiv 1 \bmod (4)$. Given such subsets $A$ and $B$ of $\mathbb{F}_{q}$, let $S$ be the set of all $x \in \mathbb{F}_{q^{\prime}}$ satisfying the above condition. Let $C:=A \cup B$, let $n=|C|$, and let $\chi: \mathbb{F}_{q^{\prime}} \rightarrow \mathbb{C}$ be the quadratic residue character of $\mathbb{F}_{q^{\prime}}$, defined by $\chi(x)=1,-1$ or 0 as $x$ is a quadratic residue, a non-residue or 0 . Note that $\chi(x y)=\chi(x) \chi(y)$ for all $x, y \in \mathbb{F}_{q^{\prime}}$.

For each $x \in \mathbb{F}_{q^{\prime}} \backslash C$ we have

$$
\prod_{a \in A}(1+\chi(x-a)) . \prod_{b \in B}(1-\chi(x-b))= \begin{cases}2^{n} & \text { if } x \in S \\ 0 & \text { otherwise }\end{cases}
$$

It follows that $S$ is non-empty if and only if

$$
s:=\sum_{x \notin C}\left(\prod_{a \in A}(1+\chi(x-a)) . \prod_{b \in B}(1-\chi(x-b))\right)>0 .
$$

Summing over all $x \in \mathbb{F}_{q^{\prime}}$ instead, let us define

$$
t:=\sum_{x \in \mathbb{F}_{q^{\prime}}}\left(\prod_{a \in A}(1+\chi(x-a)) . \prod_{b \in B}(1-\chi(x-b))\right) .
$$

Expanding the product on the right-hand side, we have

$$
t=\sum_{x} 1+\sum_{x} \sum_{a} \chi(x-a)-\sum_{x} \sum_{b} \chi(x-b)+\cdots,
$$


where the first term is $q^{\prime}$ and the second and third are 0 . To aid our consideration of the remaining terms, let us write $C=\left\{c_{1}, \ldots, c_{n}\right\}$. Then it follows from the above that

$\left|t-q^{\prime}\right| \leq \sum_{i_{1}<i_{2}}\left|\sum_{x} \chi\left(x-c_{i_{1}}\right) \chi\left(x-c_{i_{2}}\right)\right|+\cdots+\sum_{i_{1}<\cdots<i_{k}}\left|\sum_{x} \chi\left(x-c_{i_{1}}\right) \cdots \chi\left(x-c_{i_{k}}\right)\right|+\cdots$

where $i_{1}, \ldots, i_{k} \in\{1, \ldots, n\}$ in each case. Weil's estimate [28] for character sums implies that

$$
\sum_{x} \chi\left(x-c_{i_{1}}\right) \cdots \chi\left(x-c_{i_{k}}\right)=O\left(\sqrt{q^{\prime}}\right) \text { as } q^{\prime} \rightarrow \infty
$$

for each such $k$-tuple $\left(i_{1}, \ldots, i_{k}\right)$ (see Remark 1 for further explanation), so it follows immediately that for a given pair of sets $A$ and $B$, and thus for a fixed $n$, we have

$$
\left|t-q^{\prime}\right|=O\left(\sqrt{q^{\prime}}\right) \quad \text { as } \quad q^{\prime} \rightarrow \infty .
$$

Now

$$
t-s=\sum_{x \in C}\left(\prod_{a \in A}(1+\chi(x-a)) . \prod_{b \in B}(1-\chi(x-b))\right)
$$

depends only on the sets $A$ and $B$, and not on $q^{\prime}$, so we have $s>0$ for all sufficiently large $q^{\prime}$, as required.

\section{Remarks on the proof}

1. Weil proved in [28] that if $\chi$ is a multiplicative character of order $d$ of a finite field $\mathbb{F}_{q}$ (one whose values are the $d$ th roots of 1 in $\mathbb{C}$ ), and $f(x)$ is a polynomial of degree $k$ over $\mathbb{F}_{q}$ not of the form $c g(x)^{d}$ for any $c \in \mathbb{F}_{q}$ and $g(x) \in \mathbb{F}_{q}[x]$, then

$$
\left|\sum_{x \in \mathbb{F}_{q}} \chi(f(x))\right| \leq(k-1) \sqrt{q}
$$

(See [26, p. 53], for example.) Replacing $q$ with $q^{\prime}$, taking $\chi$ to be the quadratic residue character, which has degree $d=2$, and taking $f(x)=\left(x-c_{i_{1}}\right) \cdots\left(x-c_{i_{k}}\right)$ we obtain the estimate (5.2) used above. In fact, when $k=2$ we have an exact value in (5.2): this is Jacobsthal's Lemma [17], used by Paley in [23], which states that

$$
\sum_{x \in \mathbb{F}_{q}} \chi(x-u) \chi(x-v)=-1
$$

for all $u \neq v$ in $\mathbb{F}_{q}$. This can be proved in a few simple lines by using the substitution $w=(x-v) /(x-u)$. The parameters $\lambda=(q-5) / 4$ and $\mu=(q-1) / 4$ for the strongly regular graph $P(q)$ then follow by a simple version of the calculation used in Section 5 .

2. The argument used to prove Theorem 3.1 in fact shows that

$$
|S|=\frac{s}{2^{n}} \sim \frac{q^{\prime}}{2^{n}} \quad \text { as } q^{\prime} \rightarrow \infty, n \text { fixed }
$$


which is what one would expect for Paley graphs on heuristic grounds, regarding adjacency or non-adjacency of vertices as independent events with equiprobable outcomes. Bollobás and Thomason [3] have given a more precise estimate, equivalent in our notation to

$$
|| S\left|-\frac{q^{\prime}}{2^{n}}\right| \leq \frac{1}{2}\left(n-2+2^{1-n}\right) \sqrt{q^{\prime}}+\frac{n}{2} .
$$

3. In [2] Blass, Exoo and Harary, working with the Paley graphs $P(p)$ for primes $p \equiv 1$ mod (4), needed to show that given any integer $n \geq 1$, if $p$ is sufficiently large then for any disjoint $n$-element sets $A$ and $B$ of vertices of $P(p)$ there is a vertex $x$ adjacent to every $a \in A$ and to no $b \in B$. Their argument (based on one for tournaments by Graham and Spencer [16]) was similar to that used in Section 5, except that in place of Weil's character sum estimate for fields $\mathbb{F}_{q}$ they used one by Burgess [5], that if $p$ is prime and $c_{1}, \ldots, c_{k}$ are distinct elements of $\mathbb{F}_{p}$, then

$$
\left|\sum_{x \in \mathbb{F}_{p}} \chi\left(x-c_{1}\right) \ldots \chi\left(x-c_{k}\right)\right| \leq(k-1) \sqrt{p}
$$

where $\chi$ is the quadratic residue character (Legendre symbol) $\bmod (p)$.

4. Chung [11] has given some generalisations of the character sum estimates by Weil and Burgess, with applications to the discrepancy of finite graphs, including the Paley graphs; for any graph this is the maximum, over all $s$, of the difference between the maximum number of edges of an $s$-vertex subgraph and the average for that $s$. Estimating character sums is a major activity; Paley himself was an early contributor in [22], but this was in connection with number theory (specifically Dirichlet series), not graph theory.

5. For prime powers $q \equiv-1 \bmod (4)$ the construction in Section 2 yields the Paley tournament $T(q)$, a complete graph $K_{q}$ with directed edges, and the construction in Section 3 yields, for each prime $p \equiv-1 \bmod (4)$ and infinite set $E$ satisfying the conditions given there, an infinite Paley tournament $T\left(p^{E}\right)$. Again, there are uncountably many of these objects, but a slight adaptation of the preceding arguments shows that they are all isomorphic to the countable random tournament; a model of this can be obtained by applying the construction in Section 4 to primes $p, q \equiv-1 \bmod (4)$, where quadratic reciprocity now gives $\left(\frac{p}{q}\right)\left(\frac{q}{p}\right)=-1$.

6. Peter Cameron [9] has suggested a more general construction using ultraproducts of finite fields, rather than direct limits, together with Łośs Theorem, to approximate the random graph (see also [20, Example 1.3.6], based on asymptotic classes and ultraproducts); this has the advantage of allowing finite fields of different characteristics to be used, thus yielding fields of characteristic 0 .

\section{Automorphism groups}

It follows from a theorem of Carlitz [10] that the automorphism group Aut $P(q)$ of $P(q)$ is the subgroup $A \Delta L_{1}(q)$ of index 2 in $A \Gamma L_{1}(q)$ consisting of the transformations

$$
t \mapsto a t^{\gamma}+b \quad\left(a, b \in \mathbb{F}_{q}, \chi(a)=1, \gamma \in \mathrm{Gal} \mathbb{F}_{q}\right)
$$

of the vertex set $\mathbb{F}_{q}$, where Gal $\mathbb{F}_{q}$ is the Galois group or automorphism group of $\mathbb{F}_{q}$, a cyclic group of order $\log _{p} q$ generated by the Frobenius automorphism $t \mapsto t^{p}$. The affine 
transformations (those elements with $\gamma=1$ ) and the translations (those with $\gamma=1$ and $a=1$ ) form normal subgroups $A H L_{1}(q)$ (' $H$ ' for 'half') and $T_{1}(q)$ of $A \Delta L_{1}(q)$ with

$$
A \Delta L_{1}(q) \geq A H L_{1}(q)>T_{1}(q)>1,
$$

and the abelian quotients in this series show that $A \Delta L_{1}(q)$ is solvable, of derived length at most 3 .

One might hope that the automorphism group of $P\left(p^{r E}\right)$ for $r=1$ or 2 would have a similar structure. Clearly it contains the subgroup $A \Delta L_{1}\left(p^{r E}\right)$ of index 2 in $A \Gamma L_{1}\left(p^{r E}\right)$ consisting of the transformations

$$
t \mapsto a t^{\gamma}+b \quad\left(a, b \in \mathbb{F}_{p^{r E}}, \chi(a)=1, \gamma \in \mathrm{Gal} \mathbb{F}_{p^{r E}}\right) .
$$

Here Gal $\mathbb{F}_{p^{r E}}$ is not the direct limit of the groups Gal $\mathbb{F}_{p^{r e}}$ for $e \in E$, but their inverse limit: this can be identified with the (uncountable) subgroup of the cartesian product $\prod_{e \in E} \mathrm{Gal} \mathbb{F}_{p^{r e}}$ consisting of those elements whose coordinates $\gamma_{r e} \in \mathrm{Gal} \mathbb{F}_{p^{r e}}$ are consistent with the restriction mappings Gal $\mathbb{F}_{p^{r f}} \rightarrow \mathrm{Gal} \mathbb{F}_{p^{r e}}$ induced by inclusions $\mathbb{F}_{p^{r e}} \subseteq \mathbb{F}_{p^{r f}}$ for $e$ dividing $f \in E$.

As in the finite case, this group $A \Delta L_{1}\left(p^{r E}\right)$ is solvable, of derived length 3 . However, the facts that $P\left(p^{r E}\right) \cong R$ and that Aut $R$ acts transitively on isomorphism classes of finite induced subgraphs of $R$ (by the back-and-forth argument used in Section 4) destroy any hope that this subgroup might be the whole of Aut $P\left(p^{r E}\right)$. Indeed, far from being solvable, Aut $R$ has been shown by Truss [27] to be a simple group, and to contain a subgroup isomorphic to the symmetric group on a countably infinite set.

\section{Generalised Paley graphs}

In 2009 Lim and Praeger [19] introduced generalised Paley graphs $P_{d}(q)$, where $q$ is a prime power $p^{e}$ and $d$ divides $q-1$ (for convenience, we have changed their notation). Again the vertex set is $\mathbb{F}_{q}$, but now vertices $x$ and $y$ are adjacent if and only if $x-y$ is contained in the unique subgroup $D$ of index $d$ in the multiplicative group $\mathbb{F}_{q}^{*}$, consisting of the non-zero $d$ th powers. To give an undirected graph we assume that if $q$ is odd then the order $(q-1) / d$ of $D$ is even. For example, taking $d=2$ gives the Paley graphs $P(q)=P_{2}(q)$.

The construction in Section 3 carries through in the obvious way to give infinite generalised Paley graphs $P_{d}\left(p^{r E}\right)$ where $r$ is the multiplicative order of the prime $p$ mod $(2 d)$ (or $\bmod (d)$ if $p=2$ ), except that we now need $E$ to consist of integers $e$ coprime to $d$. The proof of Theorem 3.1 also carries through, provided we take $\chi$ to be a multiplicative character of $\mathbb{F}_{q^{e}}$ of degree $d$ (equivalently with kernel $D$ ), and replace the factor $1+\chi(x-a)$ in equation (5.1) with

$$
1+\chi(x-a)+\chi(x-a)^{2}+\cdots+\chi(x-a)^{d-1}=\prod_{j=1}^{d-1}\left(\chi(x-a)-\omega^{j}\right)
$$

where $\omega$ is a primitive $d$ th root of 1 in $\mathbb{C}$; again we can apply Weil's estimate, now in the more general form (6.1) given in Remark 1, to show that $P_{d}\left(p^{r E}\right) \cong R$.

The remarks in Section 7 about automorphism groups also apply here, though it should be noted that, as shown in [19], there are examples where $d$ does not divide $p-1$ and Aut $P_{d}(q)$ is significantly larger than the obvious analogue of $A \Delta L_{1}(q)$. 


\section{Symmetry versus asymmetry}

The main aim of Erdős and Rényi in [14] was to consider, in the contexts of finite and countably infinite graphs, the balance between symmetric and asymmetric graphs, those with and without a non-identity automorphism. Most of the paper concerns finite graphs, and here they proved, in a very precise sense, that not only are most graphs asymmetric, but in fact they are on average a long way from being symmetric. For a finite graph $G=(V, E)$ they defined $A(G)$ to be the least number of edge-changes (insertions or deletions) required to convert $G$ into a symmetric graph on $V$. We may identify $G$ with its edge set $E$, regarded as an element of the power set $\mathcal{P}\left(V^{(2)}\right)=\left(\mathbb{F}_{2}\right)^{V^{(2)}}$ of the set $V^{(2)}$ of 2-element subsets of $V$; the Hamming distance between two graphs $(V, E)$ and $\left(V, E^{\prime}\right)$, with respect to the basis consisting of the graphs with one edge, is $\left|E \oplus E^{\prime}\right|$ where $\oplus$ denotes symmetric difference, so $A(G)$ is the distance from $G$ to the nearest symmetric graph on $V$.

For distinct vertices $u$ and $v$ in $G$ Erdôs and Rényi defined $\Delta_{u v}$ to be the number of vertices $w \neq u, v$ adjacent to just one of $u$ and $v$. By making $\Delta_{u v}$ edge-changes one can give $u$ and $v$ the same neighbours, allowing an automorphism transposing them and fixing all other vertices, so

$$
A(G) \leq \min _{u \neq v} \Delta_{u v}
$$

By a simple counting argument they showed that if $G$ has order $n$ then

$$
\min _{u \neq v} \Delta_{u v} \leq\left\lfloor\frac{n-1}{2}\right\rfloor
$$

so that

$$
A(G) \leq\left\lfloor\frac{n-1}{2}\right\rfloor .
$$

They then showed that 'most' graphs $G$ of order $n$ have $A(G)$ close to $\lfloor(n-1) / 2\rfloor$, so that they are very far from being symmetric. As an aside they defined a $\Delta$-graph to be one achieving equality in (9.1), and noted that the graphs $P(q)$ have this property: indeed, $\Delta_{u v}=(q-1) / 2$ for all pairs $u \neq v$ in $P(q)$, another simple consequence of Jacobsthal's Lemma. Of course, these graphs are exceptional from this point of view, in that they satisfy $A(P(q))=0$.

By contrast, Erdős and Rényi showed in the last part of their paper that 'most' countably infinite graphs are symmetric. Indeed, it follows from their construction of $R$ and the alternative one due to Rado [24] that most such graphs are isomorphic to $R$ and are therefore highly symmetric: for example, Aut $R$ acts transitively on isomorphic finite induced subgraphs, and hence has rank 3 on the vertices. In fact, one can show that this group is uncountable, for instance by choosing a prime $p \equiv 1 \bmod (4)$ and taking $E=\left\{q^{n} \mid n \geq 1\right\}$ in Section 3 for some odd prime $q$, so that by our remarks in Section 7 Aut $R$ contains a copy of

$$
\operatorname{Gal} P\left(p^{E}\right)=\lim _{\leftarrow} \operatorname{Gal} P\left(p^{q^{n}}\right) \cong \lim _{\leftarrow} \mathbb{Z} / q^{n} \mathbb{Z} \cong \mathbb{Z}_{q}
$$

the uncountable group of $q$-adic integers.

\section{ORCID iDs}

Gareth A. Jones (D) https://orcid.org/0000-0002-7082-7025 


\section{References}

[1] O. Angel and Y. Spinka, Geometric random graphs on circles, 2019, https : / / arxiv . org / abs/1912.06770.

[2] A. Blass, G. Exoo and F. Harary, Paley graphs satisfy all first-order adjacency axioms, J. Graph Theory 5 (1981), 435-439, doi:10.1002/jgt.3190050414.

[3] B. Bollobás and A. Thomason, Graphs which contain all small graphs, European J. Combin. 2 (1981), 13-15, doi:10.1016/s0195-6698(81)80015-7.

[4] A. Brouwer, Paley graphs, https://www.win.tue.nl/ aeb/graphs/Paley. html.

[5] D. A. Burgess, On character sums and primitive roots, Proc. London Math. Soc. (3) 12 (1962), 179-192, doi:10.1112/plms/s3-12.1.179.

[6] P. J. Cameron, Oligomorphic permutation groups, volume 152 of London Mathematical Society Lecture Note Series, Cambridge University Press, Cambridge, 1990, doi:10.1017/ cbo9780511549809.

[7] P. J. Cameron, The random graph, in: The mathematics of Paul Erdös, II, Springer, Berlin, volume 14 of Algorithms Combin., pp. 333-351, 1997, doi:10.1007/978-3-642-60406-5_32.

[8] P. J. Cameron, The random graph revisited, in: European Congress of Mathematics, Vol. I (Barcelona, 2000), Birkhäuser, Basel, volume 201 of Progr. Math., pp. 267-274, 2001, doi: 10.1007/978-3-0348-8268-2_15.

[9] P. J. Cameron, Email to the author, 112019.

[10] L. Carlitz, A theorem on permutations in a finite field, Proc. Amer. Math. Soc. 11 (1960), 456459, doi:10.2307/2034798, errata ibid. 999-1000.

[11] F. R. K. Chung, Several generalizations of Weil sums, J. Number Theory 49 (1994), 95-106, doi:10.1006/jnth.1994.1084.

[12] P. Dembowski, Finite geometries, Ergebnisse der Mathematik und ihrer Grenzgebiete, Band 44, Springer-Verlag, Berlin-New York, 1968, https://www.springer.com/gp/book/ 9783540617860.

[13] J. D. Dixon and B. Mortimer, Permutation groups, volume 163 of Graduate Texts in Mathematics, Springer-Verlag, New York, 1996, doi:10.1007/978-1-4612-0731-3.

[14] P. Erdős and A. Rényi, Asymmetric graphs, Acta Math. Acad. Sci. Hungar. 14 (1963), 295-315, doi:10.1007/bf01895716.

[15] M. Farber, L. Mead and L. Strauss, The Rado simplicial complex (2019), https : / / arxiv . org/abs/1912.02515.

[16] R. L. Graham and J. H. Spencer, A constructive solution to a tournament problem, Canad. Math. Bull. 14 (1971), 45-48, doi:10.4153/cmb-1971-007-1.

[17] E. Jacobsthal, Über die Darstellung der Primzahlen der Form $4 n+1$ als Summe zweier Quadrate, J. Reine Angew. Math. 132 (1907), 238-246, doi:10.1515/crll.1907.132.238.

[18] G. A. Jones, Paley and the Paley graphs, in: Isomorphisms, Symmetry and Computations in Algebraic Graph Theory, WAGT 2016, Springer Proceedings in Mathematics \& Statistics, volume 305, pp. 155-183, 2020, doi:10.1007/978-3-030-32808-5_5.

[19] T. K. Lim and C. E. Praeger, On generalized Paley graphs and their automorphism groups, Michigan Math. J. 58 (2009), 293-308, doi:10.1307/mmj/1242071694.

[20] D. Macpherson and C. Steinhorn, Definability in classes of finite structures, in: Finite and algorithmic model theory, Cambridge Univ. Press, Cambridge, volume 379 of London Math. Soc. Lecture Note Ser., pp. 140-176, 2011, doi:10.1017/cbo9780511974960.005. 
[21] D. Marker, Model Theory: An Introduction, volume 217 of Graduate Texts in Mathematics, Springer-Verlag, New York, 1st edition, 2002, https://www.springer.com/gp/ book/9780387987606.

[22] R. E. A. C. Paley, A Theorem on Characters, J. London Math. Soc. 7 (1932), 28-32, doi: 10.1112/jlms/s1-7.1.28.

[23] R. E. A. C. Paley, On orthogonal matrices, Journal of Mathematics and Physics 12 (1933), 311-320, doi:10.1002/sapm1933121311.

[24] R. Rado, Universal graphs and universal functions, Acta Arith. 9 (1964), 331-340, doi:10.4064/ aa-9-4-331-340.

[25] H. Sachs, Über selbstkomplementäre Graphen, Publ. Math. Debrecen 9 (1962), 270-288.

[26] W. M. Schmidt, Equations over finite fields. An elementary approach, Lecture Notes in Mathematics, Vol. 536, Springer-Verlag, Berlin-New York, 1976, doi:10.1007/bfb0080437.

[27] J. K. Truss, The group of the countable universal graph, Math. Proc. Cambridge Philos. Soc. 98 (1985), 213-245, doi:10.1017/s0305004100063428.

[28] A. Weil, Numbers of solutions of equations in finite fields, Bull. Amer. Math. Soc. 55 (1949), 497-508, doi:10.1090/s0002-9904-1949-09219-4. 